\title{
Some Group Runs Based Multivariate Control Charts for Monitoring the Process Mean Vector
}

\author{
Mukund Parasharam Gadre1, Vikas Chintaman Kakade² \\ ${ }^{1}$ Department of Statistics, Savitribai Phule Pune University, Pune, India \\ ${ }^{2}$ Department of Statistics, Tuljaram Chaturchand College, Baramati, India \\ Email:drmukund.gadre@gmail.com,vikas.c.kakade@gmail.com
}

How to cite this paper: Gadre, M.P. and Kakade, V.C. (2016) Some Group Runs Based Multivariate Control Charts for Monitoring the Process Mean Vector. Open Journal of Statistics, 6, 1098-1109. http://dx.doi.org/10.4236/ojs.2016.66089

Received: October 10, 2016

Accepted: December 10, 2016

Published: December 14, 2016

Copyright $\odot 2016$ by authors and Scientific Research Publishing Inc. This work is licensed under the Creative Commons Attribution International License (CC BY 4.0).

http://creativecommons.org/licenses/by/4.0/

\begin{abstract}
In this article, we propose two control charts namely, the "Multivariate Group Runs' (MV-GR-M)" and the "Multivariate Modified Group Runs' (MV-MGR-M)" control charts, based on the multivariate normal processes, for monitoring the process mean vector. Methods to obtain the design parameters and operations of these control charts are discussed. Performances of the proposed charts are compared with some existing control charts. It is verified that, the proposed charts give a significant reduction in the out-of-control "Average Time to Signal" (ATS) in the zero state, as well in the steady state compared to the Hotelling's $T^{2}$ and the synthetic $T^{2}$ control charts.
\end{abstract}

\section{Keywords}

CRL Chart, Hotelling's $T^{2}$ Chart, Synthetic $T^{2}$ Chart, Average Time to Signal (ATS), Zero State ATS, Average Run Length (ARL)

\section{Introduction}

In many industrial processes, quality of the product may depend on two or more quality characteristics (may be dependent), which need to be controlled and monitored simultaneously. In the last decade or two, various multivariate procedures have been developed for simultaneous monitoring of such characteristics. Most of these procedures are to detect shifts in the process mean vector. In such a case, data in terms of vectors follow $p$-variate normal distribution with mean vector $\mu$ and covariance matrix $\boldsymbol{\Sigma}$. Hotelling, $\mathrm{H}$. [1] introduced the Hotelling's $T^{2}$ control chart which is used to monitor the multivariate process and its operation is based only on the most recent observation, therefore it is insensitive to detect small and moderate shifts in the mean vector. To overcome this drawback during the last decade, improvement of the Hotelling's $T^{2}$ sta- 
tistic has attracted for the research work.

$\mathrm{Wu}$ and Spedding [2] developed the synthetic $\bar{X}$ chart as a combination of the Shewhart $\bar{X}$ chart and the "Conforming Run Length" (CRL) chart for detecting shifts in the process mean. The CRL chart is an attribute control chart proposed by Bourke [3] for monitoring fraction nonconforming. It was shown that the synthetic $\bar{X}$ chart outperforms the Shewhart $\bar{X}$ chart over the entire range of shifts in the process mean. The development of the synthetic control chart for a univariate process has been also documented by Calzadaand Scariano [4], Davis and Woodall [5], Scariano and Calzada [6], Huang and Chen [7], and Costa and Rahim [8]. The development of the Multivariate synthetic control chart for monitoring process Mean vector (MV-Syn-M) has been proposed by Ghute and Shirke [9]. This chart is developed as a combination of the Hotelling's $T^{2}$ chart and the CRL chart. The MV-Syn-M chart is an extension of the synthetic chart in multivariate normal data.

Purpose of this article is to improve the efficiency of the Hotelling's $T^{2}$ chart and the "Multivariate Synthetic control chart to detect shifts in the Mean vector" (MV-Syn-M) by using the recently developed concept of "Group Runs" (GR) and the "Modified Groups Runs" (MGR) control charts. The development of the GR control chart [10] and MGR control chart [11] for univariate process has been documented by Gadre and Rattihalli. We propose the "Multivariate Group Runs control chart for Mean vector" (MV-GR-M) and the "Multivariate Modified Group Runs control chart for Mean vector" (MV-MGR-M) charts, which detect process changes faster than the Hotelling's $T^{2}$ chart and the MV-Syn-M chart.

Description of the related multivariate control charts for the mean vector is given in Section 2. Section 3, includes the description and design of the GR and MGR charts. Description of the runs rule representation of the MV-GR-M and MV-MGR-M charts is given in Section 4. In the subsequent section, it is illustrated that in the zero state, MV-GR-M and MV-MGR-M charts outperform as compared to the Hotelling's $T^{2}$ chart and the MV-Syn-M chart. We also give one real life situation for the effectiveness of the MV-GR-M and the MV-MGR-M charts. In Section 6, the steady state performances of the MV-GR-M and the MV-MGR-M charts are studied. Concluding remarks are given in the last section.

\section{Some Multivariate Control Charts for the Process Mean}

This section briefly describes some multivariate control charts to monitor the mean vector of a multivariate normally distributed process namely, Hotelling's $T^{2}$ chart and the MV-Syn-M chart.

Let $\boldsymbol{X}_{1}, \boldsymbol{X}_{2}, \cdots, \boldsymbol{X}_{n}$ be a random sample from $N_{p}(\boldsymbol{\mu}, \Sigma)$ distribution. Here, $\boldsymbol{\mu}$ is the process mean vector and $\Sigma$ is the process covariance matrix. These vectors represent measurements of $p$ quality characteristics. Let $\underline{\bar{X}}$ be the sample mean vector of the above sample and $\mu_{0}, \Sigma_{0}$ be the in control mean vector and covariance matrix respectively. The problem of interest is to detect the shift in the mean vector $\mu$. The hypothesis testing problem is equivalent to test the null hypothesis $H_{0}: \mu=\mu_{0}$ against $H_{1}: \mu \neq$ 
$\mu_{0}$. The test statistic for testing $H_{\mathrm{o}}$ against $H_{1}$ is given by

$$
T^{2}=n\left(\underline{\bar{X}}-\mu_{0}\right)^{\prime} \sum_{0}^{-1}\left(\underline{\bar{X}}-\mu_{0}\right),
$$

\subsection{The Hotelling's $T^{2}$ Chart}

This control chart is used to detect shift in mean vector for the multivariate normal data. The upper control limit is $k=\chi_{\alpha, p}^{2}$, where $\chi_{\alpha, p}^{2}$ is upper $100 \alpha$ percentage point of chi-square distribution. If the process is in-control, a test statistic $T^{2}$ is distributed as a chi-square variate with $p$ degrees of freedom, otherwise it follows as a non-central chi-square distribution with a non-centrality parameter $\lambda^{2}$, where

$$
\lambda^{2}=n\left(\boldsymbol{\mu}-\boldsymbol{\mu}_{0}\right)^{\prime} \sum_{0}^{-1}\left(\boldsymbol{\mu}-\boldsymbol{\mu}_{0}\right)=n d^{2}
$$

and $d$ denotes a shift of magnitude in the mean vector. ATS for this control chart is

$$
\text { ATS }=\frac{n}{P},
$$

where, $P=\operatorname{Pr}\left(T^{2}>k \mid d\right)$. The on-target and off-target values of $P$ are

$$
\left.\begin{array}{l}
P_{0}=\operatorname{Pr}\left(T^{2}>k \mid d=0\right) \\
\text { and } \\
P_{1}=\operatorname{Pr}\left(T^{2}>k \mid d^{*}\right)
\end{array}\right\}
$$

where, $d^{*}$ is the magnitude considered large enough to seriously impair quality of the products.

Here, we find optimal choices of the two parameters $(n, k)$ for given $\left(p, d^{*}, \Sigma_{0}\right)$ by using "Average Time to Signal" (ATS) model

$$
\left.\begin{array}{l}
\text { Minimize } \operatorname{ATS}\left(d^{*}\right) \\
\text { Subject to the constraint } \\
\operatorname{ATS}(0) \geq \tau
\end{array}\right\}
$$

\subsection{The Multivariate Synthetic Control Chart for Mean Vector (MV-Syn-M)}

In MV-Syn-M control chart, for the above problem, Ghute and Shirke [9] computed optimum design parameters $(k, L)$ for given $\left(n, p, d^{*}, \Sigma_{0}\right)$. They obtained optimal choices of the parameters by using ARL model. Further, they have not studied steady state performance for MV-Syn-M control chart. This chart consists of two sub-charts: $T^{2}$ sub-chart and CRL sub-chart. The operation of this chart is similar to that of the synthetic control chart suggested by $\mathrm{Wu}$ and Spedding [2]. Here, we obtain optimal choices of all the three parameters $(n, k, L)$ for given $\left(p, d^{*}, \Sigma_{0}\right)$ by using ATS model given in Equation (3). ATS for MV-Syn-M chart is given by

$$
\operatorname{ATS}\left(d^{*}\right)_{s}=\frac{n}{P\left(d^{*}\right)} \frac{1}{\left(1-\left(1-P\left(d^{*}\right)\right)^{L}\right)} .
$$




\section{Some Group Runs Based Control Charts for the Process Mean}

This section briefly describes some group runs based control charts based on ATS criterion, namely the GR chart and the MGR chart. These charts give a significant reduction in out of control ATS as compared to the $\underline{\bar{X}}$ chart and the synthetic control chart.

\subsection{Group Run Control Chart for Detecting Shifts in the Process Mean}

The "Group Runs" (GR) chart proposed by Gadre and Rattihalli [10] which is a combination of the Shewhart's $\bar{X}$ chart with an extended version of sample based CRL chart. The GR chart outperforms the Shewhart's $\bar{X}$ chart and the synthetic chart. Here CRL is the number of conforming samples between two consecutive non-conforming samples including the ending non-conforming sample. For simplicity, $Y_{r}$ be the $r^{\text {th }}$ sample based CRL.

\section{Some notations for the GR chart}

1) $\delta$ : Design shift in the process mean.

2) $\operatorname{ATS}(\delta)$ : The average number of units required by the time the process has gone out of control.

3) $\delta_{1}$ : Design shift in the mean, the magnitude of which is considered large enough to seriously impair the quality of the product.

4) $L_{g}$ : Lower control limit of GR Chart.

5) $\tau$. The minimum required value of $\operatorname{ATS}(0)$.

\section{Operation of the GR chart}

Stepwise procedure of operation of the GR chart is as follows.

Step-1: Inspect $n$ units in a group.

Step-2: Declare the group as conforming or nonconforming using $\bar{X}$ sub-chart.

Step-3: A process is said to be out of control, if either $Y_{1} \leq L_{g}$ or two successive $Y_{r}^{\prime}$ s are less than or equal to $L_{g}$ for the first time.

Step-4: When the process goes out control, necessary corrective action should be taken to reset and to resume it. Once the process restarts, move to Step-1, before initializing CRL to 0 .

\section{Design of the GR Chart}

In the synthetic control chart, for the same problem, $\mathrm{Wu}$ and Spedding computed optimal design parameters $\left(k, L_{g}\right)$ for the given sample size $(n)$. In case of the GR chart, optimum choices of the three parameters $\left(n, k, L_{g}\right)$ are computed. In designing GR chart, the model is based on ATS model given in (3).

Let $P$ be the probability of the group being nonconforming. It is given by,

$$
P=P(\delta)=1-P\left(L_{\bar{x} / s}<\bar{X}<U_{\bar{x} / s} \mid \bar{X} \sim N\left(\mu_{0}+\delta \sigma, \frac{\sigma}{\sqrt{n}}\right)\right) .
$$

Here $Y_{r}(r=1,2, \cdots)$ are independently and identically distributed (i.i.d) waiting time random variables with mean $1 / P$. Therefore, if $N$ is the number of non-conforming groups observed before declaring the process has gone out of control, then $E(N)$ and $\operatorname{ATS}(\delta)_{g}$ are as follows (refer Gadre and Rattihalli [10]) 


$$
\begin{gathered}
E(N)=\frac{1}{\left(1-(1-P(\delta))^{L}\right)^{2}} . \\
\operatorname{ATS}(\delta)_{g}=\frac{n}{P(\delta)} \frac{1}{\left(1-(1-P(\delta))^{L}\right)^{2}}
\end{gathered}
$$

\subsection{Modified Group Runs Control Chart for Process Mean}

"Modified Group Runs" (MGR) chart is proposed by Gadre and Rattihalli [11]. This chart outperforms the Shewhart's $\bar{X}$ chart, the synthetic chart and the GR chart. MGR chart consists of two components. The first component examines whether the group is conforming or not by using an $\bar{X}$-based procedure to detect shifts in the process mean. The second component depends on group runs based procedure and is used to decide status of the process. This component has two levels of group inspection. In the $i^{\text {th }}(i=1,2)$ level of group inspection, they examine whether a group-based CRL is not more or more than a given number $L_{p}$ the lower limit. The procedure of MGR chart is described as follows.

$\overline{\boldsymbol{X}}$-based procedure: If $\mu_{0}$ is the target value and $\sigma$ is the process variability, the group of size $\mathrm{n}$ is declared as nonconforming if the group mean

$\bar{X} \notin\left(\mu_{0}-k \frac{\sigma}{\sqrt{n}}, \mu_{0}+k \frac{\sigma}{\sqrt{n}}\right)$.

Group runs based procedure: The group runs based procedure declares the process as out of control, if $Y_{1} \leq L_{2}$ or for some $r(>1), Y_{r} \leq L_{1}$ and $Y_{(r+1)} \leq L_{2}$, for the first time. Here, $L_{1}$ is a warning limit.

In case of MGR chart to detect shifts in the process mean, let $\operatorname{ATS}(\delta)$ be the average number of units required by MGR chart to detect a shift in the process mean from $\mu_{0}$ to $\mu_{0} \pm \delta \sigma$. Let $\delta_{1}(\neq 0)$ be a given value of the shift in the mean, the magnitude of which is considered large enough to seriously impair the quality of the product. For the given input parameters $\left(\mu_{0}, \delta_{1}, \tau\right)$, we determine values of the design parameters $\left(n, k, L_{1}, L_{2}\right)$ by using the ATS model given in Equation (3) was considered. As mentioned in Gadre and Rattihalli [11], ATS for MGR chart is

$$
\operatorname{ATS}(\delta)_{\mathrm{mg}}=\frac{n}{P} \frac{Q^{L_{2}}+1-Q^{L_{1}}}{\left(1-Q^{L_{1}}\right)\left(1-Q^{L_{2}}\right)} .
$$

\section{Multivariate Group Runs Based Control Charts for the Process Mean}

In this section, we propose two multivariate group runs based control charts for monitoring the process mean using the Hotelling's $T^{2}$ statistic, namely the MV-GR-M chart and the MV-MGR-M chart.

\subsection{The MV-GR-M Chart}

\section{Some Notations for the MV-GR-M Chart}


1) $T^{2}=$ Hotelling's $T^{2}$ statistic

2) $L_{g}=$ "Lower Control Limit" (LCL) of the MV-GR-M chart.

3) $k_{g}=$ "Upper Control Limit" (UCL) for the status of a group of the MV-GR-M chart.

\section{Implementation of MV-GR-M Chart}

Stepwise procedure for the implementation of the MV-GR-M chart is as follows:

Step-1 Inspect $n$ units in succession.

Step-2 Declare the group as conforming or non-conforming through the Hotelling's $T^{2}$ statistic. The group is classified as non-conforming when $T^{2}$ falls beyond $k_{g}$.

Step-3 A process is said to be out of control, if either $Y_{1} \leq L_{g}$ or for $r>1$, two successive $Y_{r}^{\prime}$ s, are less than or equal to $L_{g}$ for the first time.

Step-4 When the process goes out-of-control, the corrective action be taken. Once the process restarts, return to Step-1 before initializing CRL to zero.

In Shewhart type control chart, the zero state and steady state ARL performances are exactly same. However, for the group runs based control charts, the zero state and the steady state ATS performance are not same. We carry out the steady state ATS performance by using the optimal design parameters from the zero state ATS model.

Gadre and Rattihalli [10] obtained the zero-state $\operatorname{ATS}\left(\operatorname{ATS}_{g}\right)$, for the GR chart which is as given in (4). During the in-control period, $P=\alpha$ and during the out-ofcontrolperiod, $P=1-\beta$. Davis and Woodall [5] obtained the "Steady-State ATS" of the synthetic chart through a Markov chain model. We also adopted steady state approach introduced by Davis and Woodall to obtain the $\mathrm{ATS}_{g}$ for the MV-GR-M chart. We developed the MAT-LAB program to compute the probability $P$ as mentioned in Equation (2).

\subsection{The MV-MGR-M Chart}

We applied the MGR technique suggested by Gadre and Rattihalli [11] to develop the MV-MGR-M control chart to detect shifts in the process mean vector. For MV-MGR-M chart, we obtain the design parameters $\left(n, k_{\mathrm{mg}}, L_{1}, L_{2}\right)$ for given input parameters $\left(P, d^{*}, \Sigma_{0}\right)$ by using ATS model in (3) and expression for ATS given in Equation (8).

\section{Numerical Examples and Comparison in the Zero State}

To compare the ATS performance of the MV-GR-M chart and the MV-MGR-M chart with the Hotelling's $T^{2}$ chart, $T^{2}$-syn chart, we consider the sets of input parameters ( $d$, $\tau$ ), in the zero state case. A macro in MAT-LAB is developed to obtain the design parameters of the MV-GR-M chart and MV-MGR-M chart for given input parameters.

Examples Related to the MV-GR-M and MV-MGR-M Charts and Its Performance in the Zero State

Example1: The input parameters $\left(d^{*}, \tau\right)$ are

$d^{*}: 0.51 .01 .52 .02 .53 .0$

t. 2000500010,000 
Considering all possible 18 combination of the input parameters $\left(d^{*}, \tau\right)$, values of the design parameters along with respective values of $(\operatorname{ATS})_{1}\left(=\operatorname{ATS}\left(d^{*}\right)\right)$ are computed for each of the three control charts and are given in Table 1.

For multivariate normal situation, since these 18 cases cover almost all the practical situations, we conclude that $n_{\mathrm{mg}} \leq n_{g} \leq n_{s}$ and $\operatorname{ATS}\left(d^{*}\right)_{\mathrm{mg}} \leq \operatorname{ATS}\left(d^{*}\right)_{g} \leq \operatorname{ATS}\left(d^{*}\right)_{s}$.

In the zero state, computations indicate that the MV-MGR-M chart is superior in detecting shifts in the process mean vector as compared to the other two compatible MV-Syn-M chart and MV-GR-M chart.

This example shows that, not only $\operatorname{ATS}\left(d^{*}\right)_{\mathrm{mg}}$ is not more than ATS of the other two charts, but also the sample size $n_{\mathrm{mg}}$ is not exceeding the sample sizes of the remaining two charts.

Normalized ATS (normalized with respect to the MV-Syn-M chart) values are computed for $(d=0)$ to $(d=3)$. For MV-Syn-M chart the normalized ATS is always unity. The entries up to $d=1.305$ are given in Table 2. For the larger values of $d$, the values of normalized ATS for all the four charts are same as those for $d \geq 1.095$. Figure 1 is also shown below to see the ATS performance of the four charts.

It is observed that for $d \geq 0.285$, we have $\operatorname{ATS}(d)_{\mathrm{mg}}<\operatorname{ATS}(d)_{g}<\operatorname{ATS}(d)_{s}<\operatorname{ATS}(d)_{\text {HOT }}$. Thus, the MV-MGR-M chart detects a shift of any size in the multivariate normal processes, for monitoring the process mean vector earlier than the MV-HOT, MV-Syn-M

Table 1. Optimal design parameters and $\operatorname{ATS}(d)$ values of the three charts.

\begin{tabular}{ccccccccccccccc}
\hline Input Parameters & \multicolumn{3}{c}{ MV-Syn-M Chart } & \multicolumn{3}{c}{ MV-GR-M Chart } & \multicolumn{3}{c}{ MV-MGR-M Chart } \\
\hline$(d, \tau)$ & $n_{s}$ & $k_{s}$ & $L_{s}$ & ATS $(d)$ & $n_{g}$ & $k_{g}$ & $L_{g}$ & ATS $(d)$ & $n_{\mathrm{mg}}$ & $k_{\mathrm{mg}}$ & $L_{\mathrm{lmg}}$ & $L_{2 \mathrm{mg}}$ & ATS $(d)$ \\
\hline$(0.5,2000)$ & 29 & 7.00 & 3 & 45.173 & 24 & 5.85 & 3 & 38.122 & 19 & 5.91 & 1 & 5 & $\mathbf{3 4 . 0 8 8}$ \\
$(1.0,2000)$ & 9 & 8.34 & 3 & 13.763 & 8 & 6.75 & 3 & 11.436 & 6 & 6.75 & 1 & 5 & 10.234 \\
$(1.5,2000)$ & 5 & 9.00 & 3 & 6.772 & 4 & 7.30 & 3 & 5.558 & 3 & 7.25 & 1 & 5 & 4.985 \\
$(2.0,2000)$ & 3 & 9.57 & 3 & 4.051 & 2 & 7.83 & 3 & 3.335 & 2 & 7.37 & 1 & 4 & 2.987 \\
$(2.5,2000)$ & 2 & 10.02 & 3 & 2.710 & 1 & 9.09 & 5 & 2.520 & 1 & 8.32 & 1 & 7 & 2.053 \\
$(3.0,2000)$ & 1 & 11.09 & 4 & 2.152 & 1 & 8.36 & 3 & 1.587 & 1 & 7.87 & 1 & 4 & 1.438 \\
$(0.5,5000)$ & 34 & 7.87 & 3 & 51.708 & 28 & 6.48 & 3 & 43.117 & 22 & 6.47 & 1 & 5 & $\mathbf{3 8 . 6 1 6}$ \\
$(1.0,5000)$ & 11 & 9.15 & 3 & 15.370 & 9 & 7.38 & 3 & 12.617 & 7 & 7.30 & 1 & 5 & $\mathbf{1 1 . 3 5 0}$ \\
$(1.5,5000)$ & 5 & 10.02 & 3 & 7.447 & 4 & 8.00 & 3 & 6.046 & 3 & 8.06 & 1 & 6 & $\mathbf{5 . 5 3 6}$ \\
$(2.0,5000)$ & 3 & 10.58 & 3 & 4.432 & 2 & 8.94 & 4 & 3.710 & 2 & 8.21 & 1 & 5 & 3.255 \\
$(2.5,5000)$ & 2 & 11.03 & 3 & 2.961 & 1 & 10.04 & 6 & 2.964 & 1 & 9.08 & 1 & 8 & $\mathbf{2 . 3 1 9}$ \\
$(3.0,5000)$ & 1 & 12.32 & 5 & 2.535 & 1 & 9.46 & 4 & 1.746 & 1 & 8.71 & 1 & 5 & $\mathbf{1 . 5 5 3}$ \\
$(0.5,10,000)$ & 38 & 8.53 & 3 & 56.572 & 31 & 6.95 & 3 & 46.739 & 25 & 6.88 & 1 & 5 & $\mathbf{4 2 . 0 1 5}$ \\
$(1.0,10,000)$ & 12 & 9.82 & 3 & 16.545 & 9 & 7.91 & 3 & 13.447 & 8 & 7.53 & 1 & 4 & $\mathbf{1 2 . 1 9 5}$ \\
$(1.5,10,000)$ & 6 & 10.58 & 3 & 7.974 & 5 & 8.36 & 3 & 6.497 & 4 & 8.03 & 1 & 4 & $\mathbf{5 . 8 5 6}$ \\
$(2.0,10,000)$ & 3 & 11.34 & 3 & 4.794 & 3 & 8.75 & 3 & 3.840 & 2 & 8.71 & 1 & 5 & $\mathbf{3 . 4 8 9}$ \\
$(2.5,10,000)$ & 2 & 11.78 & 3 & 3.194 & 2 & 9.05 & 3 & 2.544 & 1 & 9.66 & 1 & 9 & $\mathbf{2 . 5 5 6}$ \\
$(3.0,10,000)$ & 1 & 13.27 & 6 & 2.899 & 1 & 9.98 & 4 & 1.890 & 1 & 9.21 & 1 & 5 & $\mathbf{1 . 6 5 0}$ \\
\hline & & & & & & & & & & & &
\end{tabular}


Table 2. Normalised ATS values for four control charts for various values of $d$.

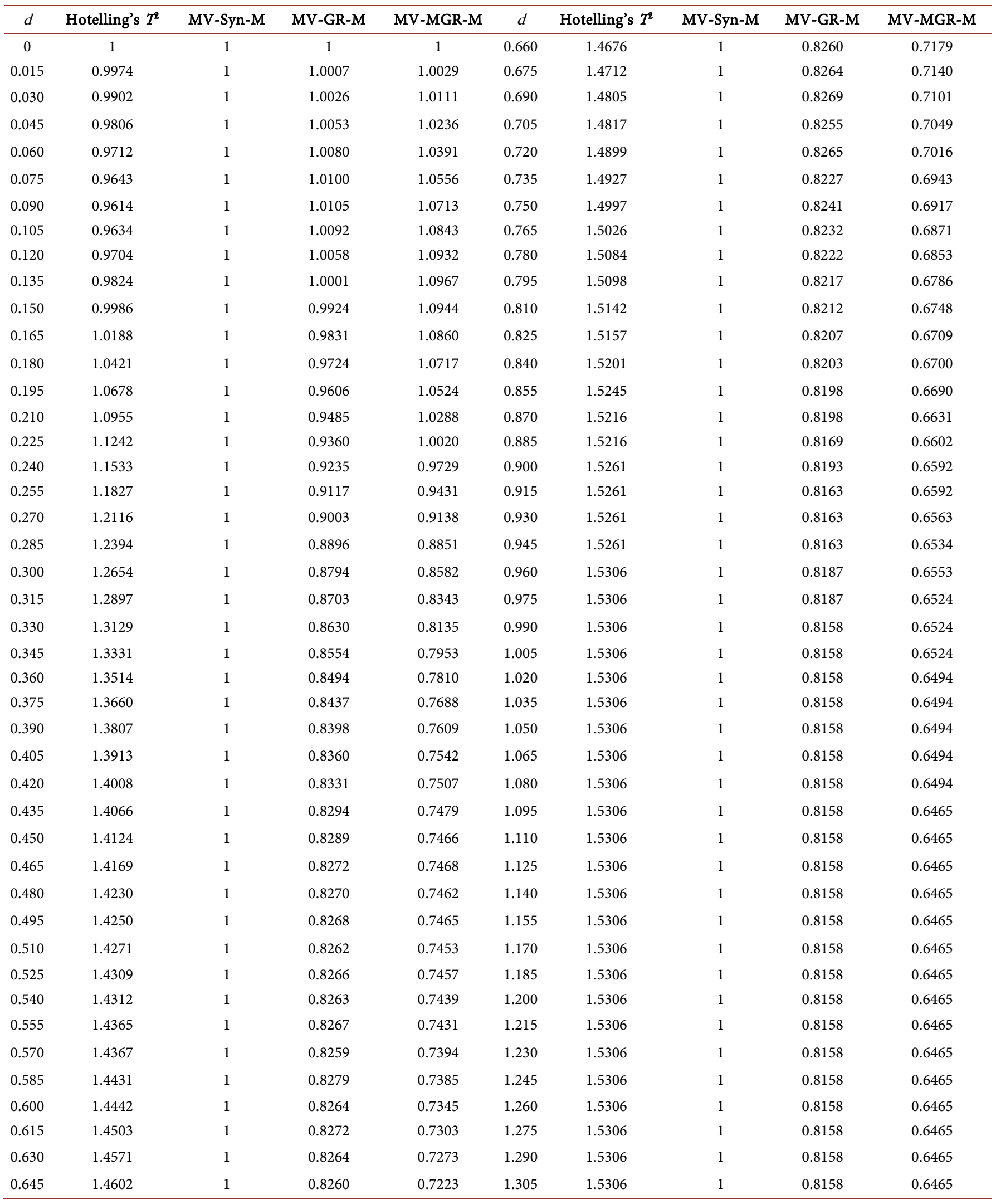




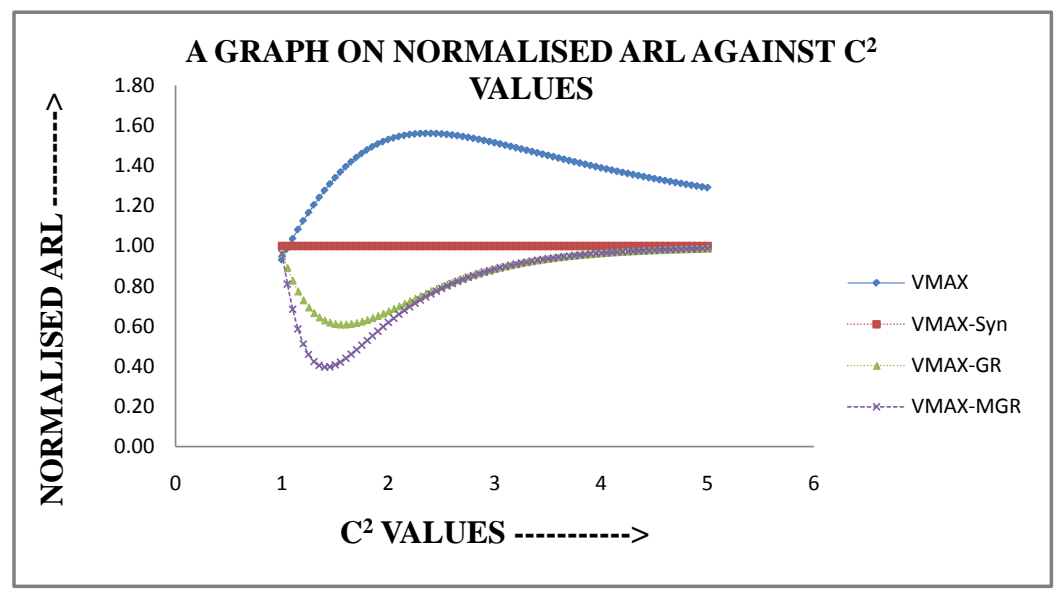

Figure 1. A graph of normalised ATS against $C^{2}$ values.

and MV-GR-M charts, though optimum values of the design parameters are calculated for a specific $d$ value.

It is to be noted that the run length based charts are not having single initial state. Therefore, it is necessary to study their performance in steady state and should be compared with that of the compatible charts. In the following section we study such performance of the MV-GR-M and MV-MGR-M charts.

\section{A real life example}

This example is given to illustrate the use of the proposed chart and compare it to the available Hotelling's $T^{2}$ and MV-Syn-M control charts. The data set is collected by the students of M.Sc. Statistics for their project. The data are from most important part, caliper of the brake system that measured the Lug-hole CD which is distance from two bottom holes of the caliper $\left(X_{1}\right)$ with the specification $142.05 \pm 0.75 \mathrm{~mm}$ and diameter which is the distance of center hole $\left(X_{2}\right)$ with the specification of $51.07 \pm 0.15 \mathrm{~mm}$ for 20 samples each size 10. According to historical information about this type of Caliper, the in-control mean vector and covariance matrix were taken as:

$$
\mu_{0}=\left(\begin{array}{c}
51.07 \\
142.05
\end{array}\right), \sum_{0}=\left(\begin{array}{cc}
0.001868 & 0.000302 \\
0.000302 & 0.0681415
\end{array}\right)
$$

Assuming that the in-control process has a $N_{2}\left(\mu_{0}, \Sigma_{0}\right)$ distribution, the process is stable with respect to its mean vector. The process is assumed to be in out of control i.e. mean vector to is shifted to the magnitude $d=0.95$ and the samples are generated from $N_{2}\left(\mu_{1}, \Sigma_{0}\right)$ distribution. For monitoring the mean vector of a bivariate process, we consider $T^{2}$ as a charting statistic. Using Equation (1), the $T^{2}$ statistic for each of the 20 samples are computed and are shown in Table 3.

We compute optimal design parameters for the three control charts for $n=10, p=2$ and $\alpha=0.05$, by choosing $d^{*}=0.95$ in Table 4 .

Here, MV-Syn-M, MV-GR-M and MV-MGR-M control charts give an out-of-control signal at sample 10, 7 and 7 respectively. This example illustrates the effectiveness of the MV-GR-M chart and MV-MGR-M chart, compared with the MV-Syn-M chart, for detecting a change of the process mean vector. 
Table 3. $T^{2}$ values for the illustrative example.

\begin{tabular}{cccc}
\hline Sample No. & $T^{\mathbf{2}}$ & Sample No. & \multicolumn{1}{c}{$T^{\mathbf{2}}$} \\
\hline 1 & 2.2397 & 11 & 0.7828 \\
2 & 2.3294 & 12 & 4.033 \\
3 & 4.0337 & 13 & 2.8089 \\
4 & 1.649 & 14 & 4.3925 \\
5 & 2.5909 & 15 & 4.9097 \\
6 & 5.2907 & 16 & 2.8456 \\
7 & 2.852 & 17 & 0.3557 \\
8 & 3.3741 & 18 & 1.7849 \\
9 & 4.6013 & 19 & 3.1023 \\
10 & 9.8068 & 20 & 4.9902 \\
\hline
\end{tabular}

Table 4. Optimal design parameters of various control charts.

\begin{tabular}{cc}
\hline Control Chart & Optimal Design Parameters \\
\hline MV-Syn-M & $\mathrm{L}=2, \mathrm{CL}=3.603$ \\
MV-GR-M & $\mathrm{L}=2, \mathrm{CL}=2.741$ \\
MV-MGR-M & $\mathrm{L}_{1}=1, \mathrm{~L}_{2}=2, \mathrm{CL}=2.51$ \\
\hline
\end{tabular}

\section{Steady State Behavior of the Various Charts}

Davis and Woodall [5], proposed runs rule for the synthetic control chart for the steady state performance. Gadre and Rattihalli [10] considered the steady state performance of the group runs control chart for detecting shifts in the process mean. Also, Gadre and Rattihalli [11] considered the steady state performance of the MGR control charts to detect increases in fraction non-conforming and shifts in the process mean. Here, we use the same runs rule for the MV-GR-M and MV-MGR-M charts.

It is to be noted that for any run length based control chart, the steady state ATS is not smaller than the zero state ATS. If the signal depends on one point only, both ATS are the same. The performances of any two charts should be compared by making the (SSATS) ${ }_{0}$ of the two charts the same. Hence, we compute the adjusted steady state ATS of chart II with respect to the chart I as

$[\operatorname{Adj} \text { S.S. } \operatorname{ATS}(d)]_{\mathrm{II}}=\left\{[\operatorname{S.S} . \operatorname{ATS}(d)]_{\mathrm{II}} /[\text { S.S. ATS }(1)]_{\mathrm{II}}\right\}\left\{\right.$ S.S. ATS $\left.(1)_{\mathrm{I}}\right\}$. Adjusted steady state ATS values corresponding to the different values of $d$ for various charts are as follows.

\section{The Steady State Performance of MV-GR-M and MV-MGR-M Charts}

Example-1 (Cont.): The following table gives the adjusted steady state ATS values corresponding to the values of din Example-1, for all three charts.

From Table 5, we observe the following:

For $d \geq 0.3$, (Adj. SSATS $)_{\text {Hot-T }}^{2}>$ (Adj. SSATS $)_{\text {MV-Syn-M }}>$ (Adj. SSATS $)_{\text {MV-GR-M }}$

The computations indicate that for shifts $(d \geq 0.3)$ in the process level, the MV-GR-M 
chart is superior in detecting the significant shifts compare to the other two compatible Hotelling's $T^{2}$ and MV-Syn-M charts in the steady state case.

From Table 6, we observe the following:

For $d \geq 0.4$, (Adj. SSATS $)_{\text {MV-Syn-M }}>(\text { Adj. SSATS })_{\text {MV-GR-M }}>(\text { Adj. SSATS })_{\text {MV-MGR-M }}$

Table 5. SSATS and Adj. SSATS for the hotelling's $T^{2}$, MV-Syn-M chart and MV-GR-M chart.

\begin{tabular}{|c|c|c|c|c|c|}
\hline & Hotelling's $T^{2}$ & MV-Syn-M & Adj. MV-Syn-M & MV-GR-M & Adj. MV-GR-M \\
\hline & $\mathrm{n}=52$ & $\mathrm{n}=34$ & $\mathrm{n}=34$ & $\mathrm{n}=\mathbf{2 8}$ & $\mathrm{n}=\mathbf{2 8}$ \\
\hline d & $k=11.26$ & $k=7.87, L=3$ & $\mathrm{k}=7.87, \mathrm{~L}=3$ & $\mathrm{~L}=3, \mathrm{k}=6.48$ & $\mathrm{k}=6.48, \mathrm{~L}=3$ \\
\hline 0 & 5000.0 & 5346.5 & 5000.0 & 5819 & 5000.0 \\
\hline 0.1 & 2440.1 & 2777.8 & 2597.8 & 3087.5 & 2652.9 \\
\hline 0.2 & 720.2 & 784.9 & 734.0 & 850.9 & 731.1 \\
\hline 0.3 & 251.0 & 253.5 & 237.1 & 261.1 & 224.4 \\
\hline 0.4 & 117.5 & 113.6 & 106.2 & 113 & 97.1 \\
\hline 0.5 & 73.7 & 68.8 & 64.3 & 67 & 57.6 \\
\hline 0.6 & 58.3 & 52.1 & 48.7 & 49.8 & 42.8 \\
\hline 0.7 & 53.4 & 45.7 & 42.7 & 42.8 & 36.8 \\
\hline 0.8 & 52.2 & 43.3 & 40.5 & 40 & 34.4 \\
\hline 0.9 & 52.0 & 42.7 & 39.9 & 39.1 & 33.6 \\
\hline 1 & 52.0 & 42.5 & 39.7 & 38.8 & 33.3 \\
\hline 1.1 & 52.0 & 42.5 & 39.7 & 38.8 & 33.3 \\
\hline 1.2 & 52.0 & 42.5 & 39.7 & 38.8 & 33.3 \\
\hline 1.3 & 52.0 & 42.5 & 39.7 & 38.8 & 33.3 \\
\hline 1.4 & 52.0 & 42.5 & 39.7 & 38.8 & 33.3 \\
\hline
\end{tabular}

Table 6. SSATS and Adj. SSATS for the MV-Syn-M chart, MV-GR-M chart and MV-MGR-M.

\begin{tabular}{|c|c|c|c|c|c|}
\hline & MV-Syn-M & MV-GR-M & Adj. MV-GR-M & MV-MGR-M & Adj. MV-MGR-M \\
\hline & $\mathrm{n}=34$ & $\mathrm{n}=28, \mathrm{~L}=3$ & $\mathrm{n}=28, \mathrm{~L}=3$ & $\mathrm{n}=22, \mathrm{~L} 1=1, \mathrm{~L} 2=5$ & $\mathrm{n}=22, \mathrm{~L} 1=1, \mathrm{~L} 2=5$ \\
\hline $\mathrm{d}$ & $k=7.87, L=3$ & $K=6.48$ & $K=6.48$ & $K=6.47$ & $K=6.47$ \\
\hline 0 & 5346.5 & 5819 & 5346.5 & 6578.9 & 5346.5 \\
\hline 0.1 & 2777.8 & 3087.5 & 2836.8 & 3808.6 & 3095.1 \\
\hline 0.2 & 784.9 & 850.9 & 781.8 & 1134.4 & 921.9 \\
\hline 0.3 & 253.5 & 261.1 & 239.9 & 328.4 & 266.9 \\
\hline 0.4 & 113.6 & 113 & 103.8 & 125.7 & 102.2 \\
\hline 0.5 & 68.8 & 67 & 61.6 & 66.1 & 53.7 \\
\hline 0.6 & 52.1 & 49.8 & 45.8 & 44.4 & 36.1 \\
\hline 0.7 & 45.7 & 42.8 & 39.3 & 35.2 & 28.6 \\
\hline 0.8 & 43.3 & 40 & 36.8 & 31.1 & 25.3 \\
\hline 0.9 & 42.7 & 39.1 & 35.9 & 29.3 & 23.8 \\
\hline 1 & 42.5 & 38.8 & 35.6 & 28.6 & 23.2 \\
\hline 1.1 & 42.5 & 38.8 & 35.6 & 28.4 & 23.1 \\
\hline 1.2 & 42.5 & 38.8 & 35.6 & 28.3 & 23.0 \\
\hline 1.3 & 42.5 & 38.8 & 35.6 & 28.3 & 23.0 \\
\hline 1.4 & 42.5 & 38.8 & 35.6 & 28.3 & 23.0 \\
\hline
\end{tabular}


The computations indicate that for shifts $(d \geq 0.4)$ in the process level, the MV-MGR-M chart is superior in detecting shifts compared to the other two compatible MV-Syn-M and MV-GR-M charts in the steady state case.

\section{Conclusion}

The MV-GR-M and MV-MGR-M control charts have been developed for the multivariate normal processes, for monitoring the process mean vector. The ATS comparison of the MV-Syn-M chart and the MV-GR-M and MV-MGR-M charts are carried out. The comparison indicates, in the zero state as well as in the steady state, the MV-GR-M and MV-MGR-M charts outperform the chart MV-Syn-M for all the shifts considered.

\section{Acknowledgements}

The authors would like to acknowledge Ms. Sanap S. S. and Ms. Sawant V. S., Students of M.Sc. (Statistics), SPPU, Pune for providing relevant data collected for their project.

\section{References}

[1] Hotelling, H. (1947) Multivariate Quality Control Illustrated by Air Testing of Sample Bombsights. In: Eisenhart, C., Hastay, M.W. and Wallis, W.A., Eds., Techniques of Statistical Analysis, McGraw Hill, New York, 111-184.

[2] Wu, Z. and Spedding T.A. (2000) A Synthetic Control Chart for Detecting Small Shifts in the Process Mean. Journal of Quality Technology, 32, 32-38.

[3] Bourke, P.D. (1991) Detecting a Shift in Fraction Nonconforming Using Run-Length Control Charts with 100\% Inspection. Journal of Quality Technology, 23, 225-238.

[4] Calzada, M.E. and Scariano, S.M. (2001) The Robustness of the Synthetic Control Chart to Non-Normality. Communication in Statistics-Simulation and Computation, 30, 311-326.

[5] Davis, R.B. and Woodall, W.H. (2002) Evaluating and Improving the Synthetic Control Chart. Journal of Quality Technology, 34, 63-69.

[6] Scariano, S.M. and Calzada, M.E. (2003) A Note on the Lower-Sided Synthetic Chart for Exponentials. Quality Engineering, 15, 677-680. https://doi.org/10.1081/QEN-120018399

[7] Chen, F.L. and Huang, H.J. (2005) A Synthetic Control Chart for Monitoring Process Dispersion with Sample Range. International Journal Advance Manufacturing Technology, 26, 842-851. https://doi.org/10.1007/s00170-003-2010-6

[8] Costa, A.F.B. and Rahim, M.A. (2006) A Synthetic Control Chart for Monitoring the Mean and Variance. Journal of Quality in Maintenance Engineering, 12, 81-88. https://doi.org/10.1108/13552510610654556

[9] Ghute, V.B. and Shirke, D.T. (2008) A Multivariate Synthetic Control Chart for Monitoring Process Mean Vector. Communication in Statistics-Theory and Methods, 37, 2136-2148.

[10] Gadre, M.P. and Rattihalli, R.N. (2004) A Group Runs Control Chart for Detecting Shifts in the Process Mean. Economic Quality Control, 19, 29-43.

[11] Gadre, M.P. and Rattihalli, R.N. (2006) Modified Group Runs Control Charts to Detect Increases in Fraction Non Conforming and Shifts in the Process Mean. Communication in Statistics-Simulation and Computation, 35, 240. 
Submit or recommend next manuscript to SCIRP and we will provide best service for you:

Accepting pre-submission inquiries through Email, Facebook, LinkedIn, Twitter, etc. A wide selection of journals (inclusive of 9 subjects, more than 200 journals)

Providing 24-hour high-quality service

User-friendly online submission system

Fair and swift peer-review system

Efficient typesetting and proofreading procedure

Display of the result of downloads and visits, as well as the number of cited articles

Maximum dissemination of your research work

Submit your manuscript at: http://papersubmission.scirp.org/

Or contact ojs@scirp.org 